\title{
Single staff cystectomy in a low-volume center: Oncological outcomes and complications
}

Philipp Baumeister $^{1}$; Davide Galioto ${ }^{1}$; Marco Moschini ${ }^{1}$; Chiara Lonati ${ }^{1}$; Stefania Zamboni ${ }^{1}$; Luca Afferi ${ }^{1}$; Patrick Stucki ${ }^{1}$; Hansjörg Danuser ${ }^{1}$; Dirk Lehnick ${ }^{2}$; Livio Mordasini ${ }^{1}$; Agostino Mattei $^{1}$

${ }^{1}$ Department of Urology, Luzerner Kantonsspital, Lucerne, Switzerland; ${ }^{2}$ Department of Biostatistics, Luzerner Kantonsspital, Lucerne,Switzerland

Cite as: Baumeister P, Galioto D, Moschini M, et al. Single staff cystectomy in a low-volume center: Oncological outcomes and complications. Can Urol Assoc J 2021 May 11; Epub ahead of print. http://dx.doi.org/10.5489/cuaj.7171

Published online May 11, 2021

Correspondence: Dr. Philipp Baumeister, Department of Urology, Luzerner Kantonsspital, Lucerne, Switzerland; philipp.baumeister@luks.ch

$* * *$

Abstract

Introduction: Radical cystectomy (RC) with bilateral pelvic lymph node dissection (PLND) is a complex surgical procedure, associated with substantial perioperative complications. Previous studies suggested reserving it to high-volume centers in order to improve oncological and perioperative outcomes. However, only limited data exist regarding low-volume centers with highly experienced surgeons. We aimed to assess oncological and perioperative outcomes after RC performed by experienced surgeons in the low-volume center of Luzerner Kantonsspital, Lucerne, $\mathrm{CH}$.

Methods: We retrospectively analyzed data of 158 patients who underwent RC and PLND performed between 2009 and 2019 at a single low-volume center by three experienced surgeons, each having performed at least 50 RCs. Complications were graded according to the 2004 modified Clavien-Dindo grading system.

Results: A total of 110 patients (70\%) received an incontinent urinary diversion (ileal conduit or ureterocutaneostomy) and 48 patients (30\%) received a continent urinary diversion (ileal orthotopic neobladder, ureterosigmoidostomy, or Mitrofanoff pouch). Median operating time was 419 minutes (interquartile range [IQR] 346-461). Overall, at RC specimen, 71.5\% of patients had urothelial carcinoma , $12.6 \%$ squamous, $3.1 \%$ sarcomatoid, $1.2 \%$ glandular, and $0.6 \%$ small cell carcinoma. Median number of lymph nodes removed was 23 (IQR 16-29.5). Positive margins were found in eight patients (5.1\%). Overall, five-year survival rate was 52.4\%. The complication rate was 56.3\%: 143 complications were found in 89 patients, $36(22.8 \%)$ with Clavien $\geq 3$. The 30-day mortality rate was $2.5 \%$. 
Conclusions: RC could be safely performed in a low-volume center by experienced surgeons with comparable outcomes to high-volume centers.

\section{Introduction}

Radical cystectomy (RC) with bilateral pelvic lymph node dissection (PLND) followed by urinary diversion (UD) represents the gold standard treatment for non-metastatic muscle-invasive and recurrent non-muscle-invasive bladder cancer $(\mathrm{BCa})[1-3]$. $\mathrm{RC}$ is a complex surgical procedure that involves genitourinary and gastrointestinal tract, pelvic organs and lymph nodes. Although RC and UD represent well-established surgical procedures, they are still associated with substantial perioperative morbidity $(27-72 \%)$ and considerable perioperative mortality rates $(0.8-8.2 \%)$ [4].

Currently, an important concern is represented by the management of high-risk procedures (such as RC) and cancer care and whether they should be centralised in specialised high-volume hospitals[5]. To date, several studies have arbitrarily provided definitions for hospital- and surgeonvolumes [6-9]. In recent literature, the definition of high-volume hospital has been set to an amount of 50-55 RC per year [6] and the threshold value for the surgeon-volume has been set to an amount of $8 \mathrm{RC}$ per surgeon per year $[8,9]$.

However, at present only limited evidence evaluates RC outcomes in small-volume medical facilities and the existing literature on this topic is lacking in accuracy and quality of peri- and postoperative complications analyses. We aimed to contribute to the present debate about centralisation of complex surgical procedures, by analysing RC perioperative and oncological outcomes and complications over a 10-year experience in the low-volume center of Luzerner Kantonsspital, Lucerne, $\mathrm{CH}$.

\section{Methods}

\section{Study design and population}

The present study involves 158 patients who underwent open radical cystectomy (ORC) or robotassisted radical cystectomy (RARC) from January 2009 to March 2019 at our institute (Luzerner Kantonsspital, Lucerne, Switzerland). All patients who underwent RC during the last 10 years were identified using our clinic operating system and clinical, surgical and pathological data were retrospectively collected. The surgical procedures were performed by 3 experienced senior staff members (Danuser H., Mattei A. and Stucki P.), each having an experience of more than 50 RC before January 2009. Informed signed consent has been obtained from all patients involved. Patient medical records, including inpatient notes, hospital discharge letters, outpatient letters and hospital readmission records were extracted from our clinical operating system and all the medical data were reviewed for demographics (age, gender, body mass index (BMI) and American Society of Anaesthesiologists score), preoperative variables (neoadjuvant chemotherapy, staging and pathology), information about intraoperative parameters (type and technique of UD, operative time, transfusions) and perioperative outcomes (complications, hospital stay). Obesity was defined as $\mathrm{BMI} \geq 30$. The Charlson Comorbidity Index (CCI) was used to assess comorbidities. All complications were graded according to the 2004 Modified Clavien-Dindo Grading System [10]. 
Pathological data included histology, tumor grade according to 1973 and 2004/2016 World Health Organization (WHO) classification[11] and stage according to the American Joint Committee on Cancer (AJCC) Tumour, Node, Metastasis (TNM) classification ( $8^{\text {th }}$ edition). Histology included urothelial carcinoma and variant histologies, such as squamous, micropapillary, sarcomatoid, small cell carcinoma and glandular variants. We assigned the diagnosis of variant histology if the pathological report revealed any morphological features that differed from pure urothelial carcinoma, regardless the actual percentage found; therefore, each variant histology could be expressed in a pure or mixed form (when it was associated to urothelial carcinoma). Each patient was assigned a specific patient-ID and all personal data were anonymously stored in a SecuTrial database. The Swiss ethics committee approved this retrospective study.

\section{Statistical analysis}

Categorical variables were expressed as frequencies and percentages; continuous variables were reported as medians, interquartile ranges (IQR), means and standard deviation (SD). Overall survival (OS) and progression-free survival (PFS) were assessed using the Kaplan-Meier method. Statistical analyses were performed using Stata (Version 15.1, StataCorp, College Station, Texas, USA).

\section{Results}

\section{Clinicopathologic characteristics}

Demographics and clinical characteristics are summarized in Table 1. Between 2009 and 2019, 158 patients underwent RC: 72\% were men, with an overall median age of 71 years (range: 29-93). Median BMI was $26.3 \mathrm{~kg} / \mathrm{m}^{2}$ and $16.5 \%$ of patients were obese. Eighty patients $(57.1 \%)$ had ASA 2 and $55(39.3 \%)$ had $A S A \geq 3$. Overall, 113 patients were diagnosed with urothelial carcinoma, 20 squamous, 5 sarcomatoid, 2 glandular and 1 small cell carcinoma. Neoadjuvant chemotherapy was given to 16 patients $(10.1 \%)$ and preoperative intravesical instillation was performed in 25 for nonmuscle-invasive $\mathrm{BCa}(15.8 \%)$.

\section{Perioperative outcomes}

Median operating time was $419 \mathrm{~min}$ (IQR: 346-461 min) (Table 2). Most of the surgical procedure $(94.3 \%)$ consists of ORC, the remaining part of RARC. An incontinent UD was performed in 110 patients (69.6\%): 104 patients $(65.8 \%)$ received an ileal conduit and $6(3.8 \%)$ an ureterocutaneostomy; a continent UD (orthotopic neobladder, ureterosigmoidostomy or Mitrofanoff pouch) was performed in 48 patients (30.4\%): among continent UD, Studer orthotopic neobladder was performed in $32 \%$ of patients. Median hospital stay was 17 days (IQR: 13-21). Respectively, 5 $(3.2 \%)$ and $13(8.2 \%)$ patients underwent a reintervention within 30 and 90 days after RC. Most of reoperations consisted of wound or uretero-ileal anastomosis revision after dehiscence.

\section{Postoperative adverse events}

Complications are depicted in Table 3. A total of 143 complications were reported in 89 patients (56.3\%): among patients experienced complications, 105 (73.4\%) had minor complications 
(Clavien class 1 or 2 ) and 38 (26.6\%) had major complications (Clavien class $\geq 3$ ) with no intraoperative deaths.

Among major complications, the most frequent events were wound infection and wound dehiscence requiring VAC-therapy or a secondary wound closure, occurring in $5(3.2 \%)$ and 7 $(4,4 \%)$ patients, respectively; complications of uretero-ileal anastomosis (dehiscence or stenosis) were observed in 7 patients (4.4\%). Gastrointestinal major complications (including bowel obstruction and perforation) were reported in 4 patients $(2.5 \%)$, whereas heart major complications in $3(1.9 \%)$. Three patients died within the hospitalisation period because of postoperative complications (Clavien 5): acute respiratory distress syndrome (ARDS), septicaemia and aspiration pneumonia. Among minor complications, blood transfusions and urinary tract infections (including low urinary tract infections, pyelonephritis and urosepsis) represented the most common events, occurring in $29(18.4 \%)$ and $17(10.8 \%)$ patients, respectively. Gastrointestinal minor complications (including paralytic ileus, dysphagia, diarrhoea, vomiting) occurred in 9 patients $(5.7 \%)$.

Respiratory and heart minor complications occurred in 8 (5.1\%) and $3(1.9 \%)$ patients, respectively. Lower limb hypoesthesia was reported in $2.5 \%$ of cases ( 4 patients).

\section{Postoperative oncologic outcomes}

Pathological outcomes for RC are shown in Table 4. Organ-confined disease (pT $\leq 2$ and $\mathrm{pN} 0$ ) was detected in 75 patients (45\%), including 30 patients (19\%) with pT $\leq 1$ and 45 (28.5\%) with pT2, while extravesical disease ( $\mathrm{pT} \geq 3$ ) was detected in 66 patients $(41.8 \%)$, including 51 patients with pT3 and 15 with pT4. Among extravesical disease patients, positive margins were detected in 8 patients $(5.1 \%)$. PLND was performed in 136 patients $(86 \%)$ and the median number of lymph nodes removed was 23 (IQR: 16-29.5). N1 and N2 diseases were found in $16(10.1 \%)$ and 22 (13.9\%) patients, respectively. At a mean follow up time of 26.5 months (SD 29.8), the 30- and 90day mortality rates were $2.5 \%$ and $4.4 \%$, respectively. Respectively, OS and PFS were $61.9 \%$ and $54.9 \%$ at 3 years, and $52.4 \%$ and $48.6 \%$ at 5 years.

\section{Discussion}

RC and UD represent complex high-risk surgical procedures, characterized by perioperative morbidities and significant mortality. Previous studies have shown a significant discrepancy in mortality rates for complex surgical procedures performed in high- and low-volume centers, suggesting that the former have better infrastructures and more experienced surgeons, resulting in better outcomes $[4,5]$. To date, several studies have provided large RC series which analysed multiple factors potentially associated with improved postoperative outcomes and decreased complications [4, 12-14]. Hospital volume [12, 14], surgeon volume [13] and patient's distance from the hospital [12] have been taken into account by analysing their relationship to survival rates after RC. A stronger correlation has been found between improved postoperative outcomes and hospital volume, rather than other factors. Moreover, the topic of centralizing RC procedures in high-volume selected institutions has been addressed by several studies, that suggested to perform $\mathrm{RC}$ in centers with high surgical caseload $[5,15,16]$. In their systematic review Goossens et al. [5] found an inverse association between postoperative mortality and hospital volume but claimed that 
additional quality criteria, such as infrastructure and experience level, should be taken into account in speculating potential centralisation projects.

Notably, to date different criteria have been proposed to define hospital- and surgeon-volumes $[6,8,17]$. Previous studies set rather low threshold values defining high volume hospital, varying between 16-32 RC per year [8, 17]. More recently, Arora et al. [6], analysing the relationship between hospital volume and RC morbidity, suggested to increase said threshold to 50-55 RC per year. Similarly, surgeon-volume definition has not been clearly defined but recent studies have set the threshold value to an amount of $8 \mathrm{RC}$ per surgeon per year [8,9]. Considering these criteria, our hospital meets the definition of low-volume hospital, counting an average of 16 RC per year.

However, although results of large RC series are currently available, data about the safety and efficacy of RC in centers with limited caseload are lacking. Most of the available studies have analysed data from large population datasets or referral centers with high patient- and surgeonvolumes $[5,12-16]$, whereas a small number of reports deals with perioperative, oncological outcomes and complications in a low volume centers, where many RC are performed. Most of monocentric studies have focused their attention on specific surgical techniques or compared operative outcomes of different surgical approaches [18, 19]. To our knowledge the current series represents the first report of outcomes of RC performed in a low-volume center by experienced surgeons that provides a comprehensive overview of results for different surgical techniques, reflecting the reality of most institutions. This study aims to implement the currently lacking literature with new data about RC performed at a low-volume center.

We made several findings. First, we considered complications as a useful mean of evaluating surgical techniques and operating outcomes. Previous studies compared RC outcomes in different centers using the Clavien-Dindo system, showing remarkable discrepancies in quantity and severity of complications, especially between low- and high-volume hospitals.

In their systematic review, Moschini et al. [4] examined 49 contemporary series of RC, reporting a postoperative complication rate of $48.7 \%$ (range: $27.0 \%-72.5 \%$ ) for ORC and $38.4 \%$ (range: $27 \%-42 \%$ ) for RARC. In our 10-year cohort, complications were reported in 89 patients with an overall complication rate of $56.3 \%$. Although only 9 patients of this cohort underwent RARC, previous studies have shown that complication rates of ORC are comparable to those of RARC [4, 18, 20, 21]. RARC has been proven equivalent to the open approach in terms of oncological and functional outcomes, with less blood loss, fewer transfusion, shorter hospital stays and quicker recovery [21]. Mani et al.[16] stratified patients who underwent RC at the same institution in 2 different time periods. In their groups, complication rate varied from $58 \%$ to $86.1 \%$ and major complications were reported in $30 \%$ and $35.2 \%$ patients, respectively. Notably, in our cohort only $22.8 \%$ of patients had major complications with Clavien class $\geq 3$, while $33.5 \%$ had minor complications (Clavien class $\leq 2$ ). Besides, 89 patients of the present series showed no sign of complications. Our complications rates are in line with previous literature on this topic in the context of Enhanced Recovery After Surgery (ERAS) pathway, which is aimed at reducing length of stay, perioperative complications and costs. In particular, Williams et al.[22] in their systematic review reported minor and major complications of $64 \%$ and $14 \%$ at 30 -day and $53 \%$ and $24 \%$ at 90-day, respectively. 
Secondly, we focused on oncological outcomes of RC. In this regard, a minimum lymph nodes yield consisting in the removal of at least 10 lymph nodes and positive surgical margins (PSM) rate less than 10-5\% have been stated as surgical quality index [23-25]. In their systematic review Perera et al. [23] reported an association between increasing nodal counts and improved OS: nodal count $>21$ has been shown to improve 10 -year OS from $32 \%$ to $59 \%$ compared to nodal count $<10$ ( $\mathrm{p}=0.005$ ). In previous RC series, the reported PSM rates varied from $2 \%$ to $16 \%[26-31]$. In a multicentre international cohort, Novara et al.[28] found PSM in $278(6.3 \%)$ of 4,410 patients, while Dotan et al. [31] reported rates of $4.2 \%$ in a single-center series of 1,591 patients. In line with previous literature, we found PSM in 8 patients (5.1\%) with a median number of 23 lymph nodes removed (IQR: 16-29.5).

Lastly, we compared the mortality rate in our cohort to those reported in recent series of highvolume center RC. Nielsen et al. [14] made an evaluation of 30- and 90-day mortality rate for 35,055 patients who underwent RC for bladder cancer in 1,118 hospitals using the National Cancer Data Base (Maryland - USA). Mortality rates at low- and high-volume centres were compared. Overall, 30 - and 90 -day mortality rates were $2.7 \%$ and $7.2 \%: 1.9 \%$ and $5.7 \%$ among high-volume hospitals and $3.2 \%$ and $8.0 \%$ among low-volume hospitals, respectively. In their systematic review Moschini et al. [4] found an average 30-day mortality rate of 3.8\% for patients who underwent ORC, ranging from $0.8 \%$ to $8.2 \%$. The 30 - and 90 -day mortality rates observed at our low-volume center were $2.5 \%$ and $4.4 \%$, respectively. When compared with outcomes observed by Nielsen et al.[14], these rates appear to be more similar to those from high-volume hospital. The reason underlying this is probably the surgical team experience. In particular, each surgeon involved in the present study had performed more than $50 \mathrm{RC}$ before this series starting period. In addition, these 3 surgeons had previously experienced a training period in Bern led by Dr. Studer, known for the orthotopic neobladder technique that bears his name.

Our study is not devoid of limitations. First, data were collected in a retrospective design and therefore subject to bias which do not allow definitive conclusions to be drawn. Secondly, our center showed low rates of both neoadjuvant and adjuvant chemotherapy compared to standard of care; a possible explanation of these results could be sought in preexisting comorbidities of patients which did not make patients eligible to cisplatin chemotherapy. Although this limitation could frequently occur in retrospective studies, it may have affected our results, especially survival outcomes. Moreover, approximately $10 \%$ of patients have not undergone LND, possibly due to patient's comorbidities or surgeon's choice. In addition, though our study covers a wide time range, the number of patients is quite small. Finally, our cohort includes two different surgical techniques (ORC vs. RARC) which have not been separately compared.

In conclusion, we found that an experienced staff operating in a low-volume centre can produce results comparable to those obtained in high-volume centers, in terms of perioperative complications and survival. Therefore, the present study suggests that patients could safely undergo $\mathrm{RC}$ in a low-volume center provided it is performed by adequately experienced surgeons. 


\section{References}

1. Alfred Witjes J, Lebret T, Compérat EM, et al. Updated 2016 EAU Guidelines on Muscleinvasive and Metastatic Bladder Cancer. Eur Urol 2017; 71: 462-475.

2. Witjes JA, Babjuk M, Bellmunt J, et al. EAU-ESMO Consensus Statements on the Management of Advanced and Variant Bladder Cancer-An International Collaborative Multistakeholder Effort $†$ : Under the Auspices of the EAU-ESMO Guidelines Committees. Eur Urol 2020; 77: 223-250.

3. Horwich A, Babjuk M, Bellmunt J, et al. EAU-ESMO consensus statements on the management of advanced and variant bladder cancer - an international collaborative multistakeholder effort: under the auspices of the EAU and ESMO Guidelines Committees. Ann Oncol 2019; 30: 1697-1727.

4. Moschini M, Simone G, Stenzl A, et al. Critical Review of Outcomes from Radical Cystectomy: Can Complications from Radical Cystectomy Be Reduced by Surgical Volume and Robotic Surgery? Eur Urol Focus 2016; 2: 19-29.

5. Goossens-Laan CA, Gooiker GA, van Gijn W, et al. A Systematic Review and Metaanalysis of the Relationship Between Hospital/Surgeon Volume and Outcome for Radical Cystectomy: An Update for the Ongoing Debate. Eur Urol 2011; 59: 775-783.

6. Arora S, Keeley J, Patel A, et al. Defining a "High Volume" Radical Cystectomy Hospital: Where Do We Draw the Line? Eur Urol Focus 2020; 6: 975-981.

7. Casey MF, Wisnivesky J, Le VH, et al. The Relationship between Centralization of Care and Geographic Barriers to Cystectomy for Bladder Cancer. Bl Cancer 2016; 2: 319-327.

8. Mayer EK, Bottle A, Aylin P, et al. The volume-outcome relationship for radical cystectomy in England: an analysis of outcomes other than mortality. BJU Int 2011; 108: E258-E265.

9. McCabe JE, Jibawi A, Javle PM. Radical cystectomy: defining the threshold for a surgeon to achieve optimum outcomes. Postgrad Med J 2007; 83: 556-560.

10. Dindo D, Demartines N, Clavien PA. Classification of surgical complications: A new proposal with evaluation in a cohort of 6336 patients and results of a survey. Ann Surg 2004; 240: 205-213.

11. Moch H, Cubilla AL, Humphrey PA, et al. The 2016 WHO Classification of Tumours of the Urinary System and Male Genital Organs-Part A: Renal, Penile, and Testicular Tumours. Eur Urol 2016; 70: 93-105.

12. Xia L, Taylor BL, Mamtani R, et al. Associations Between Travel Distance, Hospital Volume, and Outcomes Following Radical Cystectomy in Patients With Muscle-invasive Bladder Cancer. Urology 2018; 114: 87-94.

13. Waingankar N, Mallin K, Smaldone M, et al. Assessing the relative influence of hospital and surgeon volume on short-term mortality after radical cystectomy. BJU Int 2017; 120: 239-245.

14. Nielsen ME, Mallin K, Weaver MA, et al. Association of hospital volume with conditional 90-day mortality after cystectomy: an analysis of the National Cancer Data Base. BJU Int 2014; 114: 46-55.

15. Udovicich C, Perera M, Huq M, et al. Hospital volume and perioperative outcomes for radical cystectomy: a population study. BJU Int 2017; 119: 26-32.

16. Mani J, Vallo S, Brandt M, et al. What should be the patient\&\#39;s preference regarding the choice of hospital in the case of radical cystectomy? Evaluation of early complications after open radical cystectomy in a medium and high volume setting in one hospital. Patient Prefer Adherence 2016; Volume 10: 2181-2187. 
17. Smaldone MC, Simhan J, Kutikov A, et al. Trends in regionalization of radical cystectomy in three large northeastern states from 1996 to 2009. Urol Oncol Semin Orig Investig 2013; 31: 1663-1669.

18. Muto S, Kitamura K, Ieda T, et al. A preliminary oncologic outcome and postoperative complications in patients undergoing robot-assisted radical cystectomy: Initial experience. Investig Clin Urol 2017; 58: 171.

19. Nazmy M, Yuh B, Kawachi M, et al. Early and Late Complications of Robot-Assisted Radical Cystectomy: A Standardized Analysis by Urinary Diversion Type. J Urol 2014; 191: 681-687.

20. Zamboni S, Soria F, Mathieu R, et al. Differences in trends in the use of robot-assisted and open radical cystectomy and changes over time in peri-operative outcomes among selected centres in North America and Europe: an international multicentre collaboration. BJU Int 2019; 124: 656-664.

21. Wilson TG, Guru K, Rosen RC, et al. Best Practices in Robot-assisted Radical Cystectomy and Urinary Reconstruction: Recommendations of the Pasadena Consensus Panel. Eur Urol 2015; 67: 363-375.

22. Williams SB, If TD, Cumberbatch MGK, et al. Reporting Radical Cystectomy Outcomes Following Implementation of Enhanced Recovery After Surgery Protocols : A Systematic Review and Individual Patient Data Meta-analysis. Eur Urol 2020; 78: 719-730.

23. Perera M, McGrath S, Sengupta S, et al. Pelvic lymph node dissection during radical cystectomy for muscle-invasive bladder cancer. Nat Rev Urol 2018; 15: 686-692.

24. Pruthi RS, Nielsen ME, Nix J, et al. Robotic Radical Cystectomy for Bladder Cancer: Surgical and Pathological Outcomes in 100 Consecutive Cases. J Urol 2010; 183: 510-515.

25. Skinner EC, Stein JP, Skinner DG. Surgical benchmarks for the treatment of invasive bladder cancer. Urol Oncol Semin Orig Investig 2007; 25: 66-71.

26. Hussein AA, Hashmi Z, Dibaj S, et al. Reoperations following Robot-Assisted Radical Cystectomy: A Decade of Experience. J Urol 2016; 195: 1368-1376.

27. Parekh DJ, Messer J, Fitzgerald J, et al. Perioperative Outcomes and Oncologic Efficacy from a Pilot Prospective Randomized Clinical Trial of Open Versus Robotic Assisted Radical Cystectomy. J Urol 2013; 189: 474-479.

28. Novara G, Svatek RS, Karakiewicz PI, et al. Soft Tissue Surgical Margin Status is a Powerful Predictor of Outcomes After Radical Cystectomy: A Multicenter Study of More Than 4,400 Patients. J Urol 2010; 183: 2165-2170.

29. Hellenthal NJ, Hussain A, Andrews PE, et al. Surgical Margin Status After Robot Assisted Radical Cystectomy: Results From the International Robotic Cystectomy Consortium. $J$ Urol 2010; 184: 87-91.

30. Hadjizacharia P, Stein JP, Cai J, et al. The impact of positive soft tissue surgical margins following radical cystectomy for high-grade, invasive bladder cancer. World J Urol 2009; 27: 33-38.

31. Dotan ZA, Kavanagh K, Yossepowitch O, et al. Positive Surgical Margins in Soft Tissue Following Radical Cystectomy for Bladder Cancer and Cancer Specific Survival. J Urol 2007; 178: 2308-2313. 
Figures and Tables

\begin{tabular}{|c|c|}
\hline Variable & Value \\
\hline \multicolumn{2}{|l|}{ Age (years) } \\
\hline Mean (SD) & $70(10.2)$ \\
\hline Median (range) & $71(29-93)$ \\
\hline \multicolumn{2}{|l|}{ Gender } \\
\hline Male, n. $(\%)$ & $114(72)$ \\
\hline Female, n. $(\%)$ & $44(28)$ \\
\hline \multicolumn{2}{|l|}{ Body mass index $\left(\mathrm{kg} / \mathrm{m}^{2}\right)$} \\
\hline Mean (SD) & $26.7(4.3)$ \\
\hline Median (range) & $26.3(16.26-37.28)$ \\
\hline \multicolumn{2}{|l|}{ Charlson comorbidity index, $\mathrm{n}(\%)$} \\
\hline$\leq 1$ & $110(69.6)$ \\
\hline 2 & $25(15.8)$ \\
\hline 3 & $13(8.2)$ \\
\hline$\geq 4$ & $10(6.3)$ \\
\hline \multicolumn{2}{|l|}{ ASA score, n (\%) } \\
\hline 1 & $5(3.57)$ \\
\hline 2 & $80(57.14)$ \\
\hline 3 & $54(38.57)$ \\
\hline 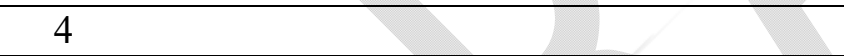 & $1(0.71)$ \\
\hline \multicolumn{2}{|l|}{ Clinical T stage, $\mathrm{n}(\%)$} \\
\hline$\leq \mathrm{cT} 2$ & $128(81)$ \\
\hline cT3 & $6(3.79)$ \\
\hline 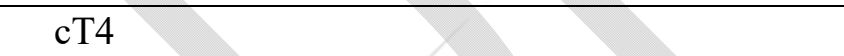 & $4(2.54)$ \\
\hline \multicolumn{2}{|l|}{ Clinical N stage, $\mathrm{n}(\%)$} \\
\hline $\mathrm{cN0}$ & $130(82.28)$ \\
\hline $\mathrm{cN1}$ & $2(1.27)$ \\
\hline $\mathrm{cN} 2$ & $5(3.16)$ \\
\hline $\mathrm{cN} 3$ & $1(0.63)$ \\
\hline \multicolumn{2}{|l|}{ Neoadjuvant chemotherapy, n (\%) } \\
\hline Yes & $16(10.1)$ \\
\hline No & $142(89.9)$ \\
\hline \multicolumn{2}{|l|}{ Adjuvant chemotherapy, $\mathrm{n}(\%)$} \\
\hline Yes & $30(18.99)$ \\
\hline No & $128(81.01)$ \\
\hline
\end{tabular}

ASA: American Society of Anesthesiologists; SD: standard deviation. 


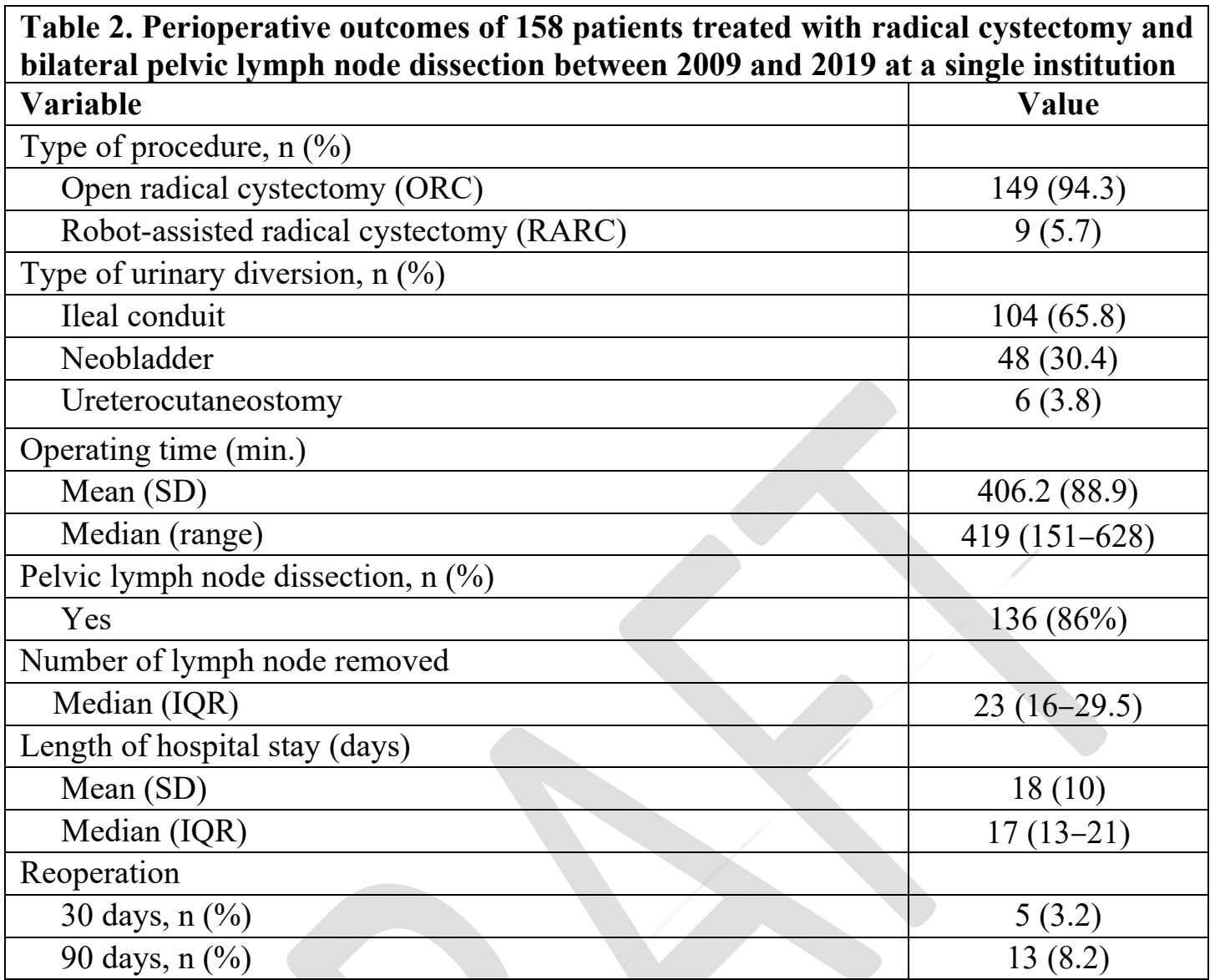

IQR: interquartile range; SD: standard deviation.

Table 3. Complications of 158 patients treated with radical cystectomy and bilateral pelvic lymph node dissection between 2009 and 2019 at a single institution

\begin{tabular}{|l|c|}
\hline Highest Clavien-Dindo class & $\mathbf{n}(\mathbf{\%})$ \\
\hline Minor complications & $53(33 \%)$ \\
\hline 1 & $7(4.4 \%)$ \\
\hline 2 & $46(29 \%)$ \\
\hline Major complications & $36(23 \%)$ \\
\hline $3 \mathrm{a}$ & $17(10 \%)$ \\
\hline $3 \mathrm{~b}$ & $14(8.9 \%)$ \\
\hline $4 \mathrm{a}$ & $2(1.3 \%)$ \\
\hline 5 & $3(1.9 \%)$ \\
\hline Overall complication rate & $89(56 \%)$ \\
\hline
\end{tabular}


Table 4. Pathological outcomes of 158 patients treated with radical cystectomy and bilateral pelvic lymph node dissection between 2009 and 2019 at a single institution

\begin{tabular}{|l|c|}
\hline Variable & Value \\
\hline Pathological T stage, $\mathrm{n}(\%)$ & $8(5.1)$ \\
\hline $\mathrm{pT} 0$ & $1(0.6)$ \\
\hline $\mathrm{pTa}$ & $3(1.9)$ \\
\hline $\mathrm{pT}$ (is & $18(11.4)$ \\
\hline $\mathrm{pT} 1$ & $45(28.5)$ \\
\hline $\mathrm{pT} 2$ & $51(32.3)$ \\
\hline $\mathrm{pT} 3$ & $15(9.5)$ \\
\hline $\mathrm{pT} 4$ & \\
\hline Pathological N stage, $\mathrm{n}(\%)$ & $101(63.9)$ \\
\hline 0 & $16(10.1)$ \\
\hline 1 & $22(13.9)$ \\
\hline 2 & $1(0.6)$ \\
\hline 3 & \\
\hline Histological variants, $\mathrm{n}(\%)$ & $113(71.5)$ \\
\hline Transitional cell carcinoma & $20(12.6)$ \\
\hline Squamous differentiation & $5(3.1)$ \\
\hline Sarcomatoid carcinoma & $2(1.2)$ \\
\hline Glandular differentiation & $1(0.62)$ \\
\hline Small cell carcinoma & $43(27)$ \\
\hline Presence of carcinoma in situ, $\mathrm{n}(\%)$ & $21(13)$ \\
\hline Lymphovascular invasion, $\mathrm{n}(\%)$ & $16(10)$ \\
\hline Perineural invasion, $\mathrm{n}(\%)$ & $8(5.1)$ \\
\hline Positive margins, $\mathrm{n}(\%)$ & \\
\hline
\end{tabular}

